\title{
Analysis of XANES spectra for tektites using machine learning algorithms
}

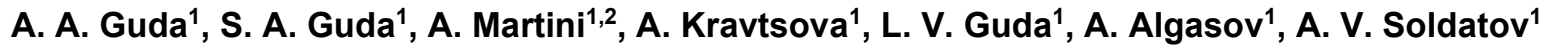 \\ ${ }^{I}$ The Smart Materials Research Institute, Southern Federal University, 344090 Rostov-on-Don, Russia, ${ }^{2}$ Department of Chemistry, \\ INSTM Reference Center and NIS and CrisDi Interdepartmental Centers, University of Torino, 10125 Torino, Italy
}

\author{
guda@sfedu.ru
}

Tektites and impactites are mainly amorphous silicate glass bodies of impact genesis. The conditions, such as pressure (P), temperature $(\mathrm{T})$, oxygen fugacity $\left(\mathrm{fO}_{2}\right)$, that existed during glass formation process influence the cation coordination. One of the common elements in impact glasses is iron, so it a useful probe of atmospheric parameters and collision conditions in geological history of Earth. X-ray absorption spectroscopy (XAS) is a direct probe of the local atomic structure around specific element applicable to liquids, single molecules or amorphous solids. Recent developments in theoretical interpretation of the X-ray absorption fine structure along with machine learning (ML) methods opened a new perspective for quantitative structural analysis of complex systems [1,2]. In this work we extend ML-driven fitting procedure to the amorphous structures of tektites, where several inequivalent sites with different coordination numbers can coexist. The analysis was performed in PyFitIt software [3]. For each possible coordination number $\mathrm{N}=2 \ldots 6$ we constructed a fragment of silica where $\mathrm{Fe}$ ion was coordinated by several $\left(\mathrm{SiO}_{4}\right)$ tetrahedral units. Structural parameters of $\mathrm{Fe}(\mathrm{SiO} 4)_{\mathrm{N}}$ cluster were varied to simulate possible bond length variation in amorphous silica both for $\mathrm{Fe}^{2+}$ and $\mathrm{Fe}^{3+}$ ions, e.g. all Fe-O distances were varied in the range [1.8 ..2.3 $\left.\AA\right]$.

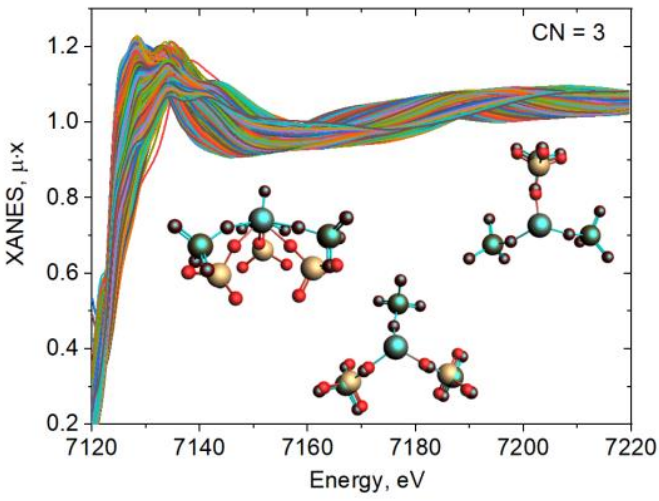

Figure 1. Theoretical training set calculated for 3-ccordinated Fe ion in $\mathrm{SiO} 2$ matrix by varying three structural parameters

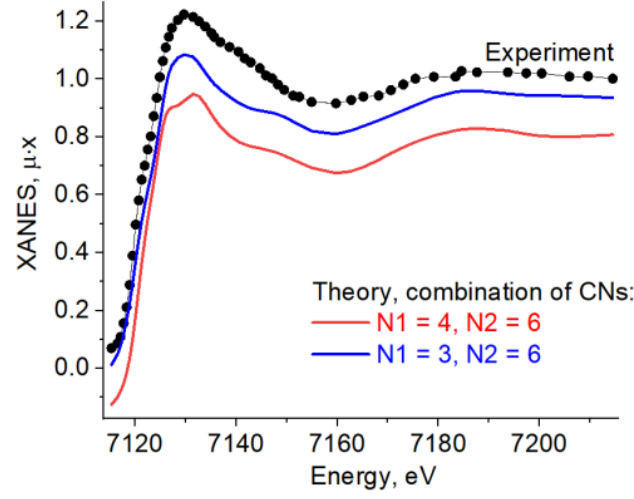

Figure 2. Best fits, obtained as a superposition of pairs with 3, 4, and 6-coordinated iron

Figure 1 shows geometry parameters for the three-coordinated Fe ions: distance to the nearest oxygen, distance to the rest two oxygens, bending angle (distortions are marked with overlay of two structures for each of three deformations). In the space of structural parameters we selected a set of 700 points where XANES spectra were calculated by means of finite difference approach implemented within FDMNES software. These spectra were used as training set for machine learning method which established the relation between spectrum and structural parameters. The best quality of approximation was achieved for Radial Basis Functions method. After algorithm was trained the PyFitIt software allows user to predict structural parameters for a given spectrum in the input (so called direct method) or predict spectrum for a given set of structural parameters (so called indirect method, the analogue of multidimensional interpolation). Figure 2 shows the analysis of the Muong-Nong tektite spectrum. The two best fits are shown as red and blue lines and use a combination of 6-coordinated iron ions either with 4-coordinated or 3-coordinated species. The resulting parameters of two fits provided average coordination number $<\mathrm{CN}>=5.4 \pm 0.2$ and average Fe-O distance $<\mathrm{R}>=1.96 \pm 0.04 \AA$ which are in a good agreement with classical EXAFS analysis.

[1] Guda, A. A., Guda, S. A., Lomachenko, K. A., Soldatov, M. A. et.al., (2019). Catalysis Today 336, 3-21.

[2] Timishenko, J, Frenkel, A. I., (2019). ACS Catal. 9, 10192-10211.

[3] Martini, A., Guda, S. A., Guda, A. A., Smolentsev, G. A., et.al., (2020). Computer Physics Communications 250, 107064

Keywords: XANES; pre-edge; radial distribution function; machine learning; radial basis functions; descriptors

This work was supported by Russian Foundation for Basic Research (project 20-32-70227).

Acta Cryst. (2021), A77, C601 